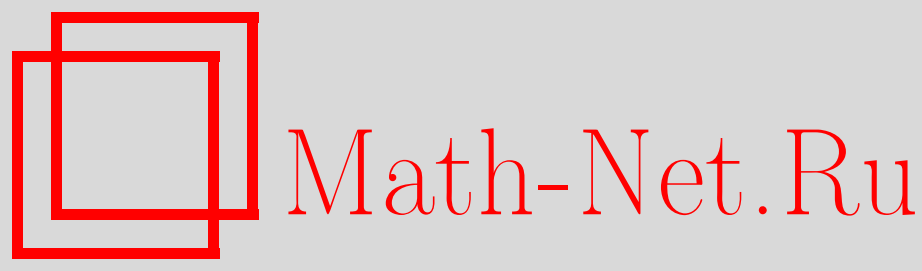

С. Фаранг-Сардруди, А. Резаи-Агдам, Л. Седжи-Гадим, Структуры Намбу на четырехмерных действительных группах Ли и связанные с ними суперинтегрируемые системы, ТMФ, 2015, том 183, номер 2, 274-300

DOI: https://doi.org/10.4213/tmf8769

Использование Общероссийского математического портала Math-Net.Ru подразумевает, что вы прочитали и согласны с пользовательским соглашением http://www . mathnet.ru/rus/agreement

Параметры загрузки:

IP : 54.92 .164 .108

26 апреля 2023 г., 16:29:02

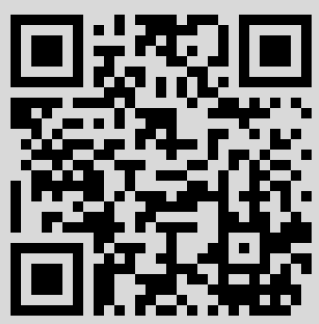




\title{
ФИЗИКА
}

Том 183, № 2

май, 2015

(C) 2015 г.

С. Фаранг-Сардруди*, А. Резаи-Агдам*, Л. Седжи-Гадим ${ }^{\dagger}$

\section{СТРУКТУРЫ НАМБУ НА ЧЕТЫРЕХМЕРНЫХ ДЕЙСТВИТЕЛЬНЫХ ГРУППАХ ЛИ И СВЯЗАННЫЕ С НИМИ СУПЕРИНТЕГРИРУЕМЫЕ СИСТЕМЫ}

\begin{abstract}
Определены все тензоры Намбу (структуры Намбу) третьего и четвертого порядков на четырехмерных действительных группах Ли. Кроме того, с помощью структур Намбу четвертого порядка получены суперинтегрируемые системы на некоторых из этих групп Ли, взятых в качестве фазовых пространств, с группами симметрий $\mathbf{A}_{4,8}$ и $\mathbf{A}_{4,10}$.
\end{abstract}

Ключевые слова: структуры Намбу, суперсимметричные системы, группы Ли.

DOI: $10.4213 / \operatorname{tmf} 8769$

\section{1. ВВЕДЕНИЕ}

В 1973 году в работе [1] Намбу изучил динамическую систему, представленную в виде гамильтоновой системы по отношению к обобщенной скобке Пуассона (скобке пуассоновского типа), введенной с помощью якобиана. Спустя годы (около двух десятилетий) Тахтаджян [2] ввел понятие структур Намбу-Пуассона (или просто структур Намбу), используя аксиоматическое определение $n$-скобки, указал основные свойства этой операции, а также дал геометрическую формулировку многообразий Намбу. Этот новый подход породил цикл статей, связанных с новыми понятиями.

Многообразие Намбу - это $C^{\infty}$-многообразие, на котором задан тензор Намбу. Тензор Намбу есть такое кососимметричное контравариантное тензорное поле на многообразии, что индуцированная скобочная операция удовлетворяет фундаментальному свойству, которое обобщает обычное тождество Якоби [3]-[7] (имеется еще одно обобщение, так называемая обобщенная скобка Пуассона [8], [9]; сравнение обоих понятий дано в работах [10], [11]). В работах [12] и [13] введено понятие группы

* Department of Physics, Azarbaijan Shahid Madani University, Tabriz, Iran. E-mail: m.farhang88@azaruniv.edu,rezaei-a@azaruniv.edu

${ }^{\dagger}$ Department of Mathematics, Azarbaijan Shahid Madani University, Tabriz, Iran. E-mail: m.sedghi88@azaruniv.edu 
Намбу-Ли. Вайсман в работе [13] распространил скобки Намбу на 1-формы и, обобщив случай Пуассона-Ли, ввел понятие групп Намбу-Ли как групп, наделенных мультипликативной структурой Намбу. Разложимость структур Намбу для групп Ли, а также соответствие между семейством левоинвариантных тензоров Намбу порядка $n$ на $m$-мерных группах Ли $G$ и семейством $n$-мерных подалгебр Ли алгебры $\mathcal{G}$ (алгебры Ли в $G$ ) было доказано Наканиши в работе [14]. В работе [15] он также ввел мультипликативные структуры Намбу на трехмерных действительных группах Ли. В настоящей статье тем же способом мы попытаемся определить мультипликативные структуры Намбу четвертого и третьего порядков на четырехмерных действительных группах Ли.

План статьи следующий. В разделе 2 для самодостаточности статьи дан обзор некоторых определений и теорем. В разделе 3 с помощью метода, примененного в работе [15], мы определим мультипликативные структуры Намбу четвертого и третьего порядков на четырехмерных действительных группах Ли. Наконец, в разделе 4, используя структуры Намбу четвертого порядка на некоторых группах Ли (т. е. на четырехмерных действительных группах Ли, имеющих симплектическую структуру [16]), мы получим суперинтегрируемые системы с указанными группами Ли в качестве фазовых пространств и группами $\mathbf{A}_{4,8}$ и $\mathbf{A}_{4,10}$ в качестве групп симметрий Ли.

\section{2. ОСНОВНЫЕ ОПРЕДЕЛЕНИЯ И ТЕОРЕМЫ}

Напомним некоторые основные определения и теоремы, связанные со структурой Намбу [12], [15]. Пусть $G-m$-мерная группа Ли с алгеброй Ли $\mathcal{G}$. Обозначим через $\Gamma\left(\Lambda^{n} T G\right)$ семейство антисимметричных $n$-векторных полей (контравариантных тензоров) на $G$. Тогда для каждого $\eta \in \Gamma\left(\Lambda^{n} T G\right)$ можно ввести $n$-скобку функций на группе Ли $G$ следующим образом:

$$
\left\{f_{1}, \ldots, f_{n}\right\}=\eta\left(d f_{1}, \ldots, d f_{n}\right), \quad f_{i} \in \mathcal{F}(G), \quad i=1, \ldots, n,
$$

где $\mathcal{F}(G)$ - алгебра $C^{\infty}$-функций на $G$. Кроме того, поскольку скобки удовлетворяют правилу Лейбница, можно определить векторное поле $X_{f_{1}, \ldots, f_{n-1}}$ как

$$
X_{f_{1}, \ldots, f_{n-1}}(g)=\left\{f_{1}, \ldots, f_{n-1}, g\right\}, \quad g \in \mathcal{F}(G) .
$$

Это векторное поле называется гамильтоновым, пространство гамильтоновых векторных полей обозначим как $\mathcal{H}$.

Введем следующие понятия [7], [14], [15].

ОПРЕДЕЛЕНИЕ 1. Элемент $\eta \in \Gamma\left(\Lambda^{n} T G\right)$ при $n \geqslant 3$ называется тензором Намбу порядка $n$, если он удовлетворяет условию $\mathcal{L}_{X_{f_{1}, \ldots, f_{n-1}}} \eta=0$ для всех $X_{f_{1}, \ldots, f_{n-1}} \in \mathcal{H}$, где $f_{i} \in \mathcal{F}(G)$; здесь $\mathcal{L}$ означает производную Ли.

ОПРЕДЕЛЕНИЕ 2. Элемент $\eta \in \Gamma\left(\Lambda^{n} T G\right)$ назовем мультипликативным тензором, если для любых $g_{1}, g_{2} \in G$ имеем

$$
\eta_{g_{1} g_{2}}=L_{g_{1 *}} \eta_{g_{2}}+R_{g_{2 *}} \eta_{g_{1}}
$$

где $R_{g_{2}}$ и $L_{g_{1}}$ - соответственно правый и левый сдвиг в $G$. Группа Ли $G$, наделенная мультипликативным тензором Намбу $\eta$, называется группой Намбу-Ли [13]. 
Следующая теорема и следствие из нее доказаны в работе [13].

Теорема 1. Пусть $G-m$-мерная группа Ли $u \mathbf{h}-n$-мерная подалгебра алгебръц $\mathcal{G}$, где $n \geqslant 3$. Для базиса $X_{1}, \ldots, X_{n}$ подалгебры $\mathbf{h}$ положим $\eta=X_{1} \wedge \cdots \wedge X_{n}$. Тогда $\eta$ - левоинвариантный тензор Намбу порядка $n$ на G. Наоборот, пусть задан левоинвариантный тензор Намбу $\eta=X_{1} \wedge \cdots \wedge X_{n} \in \Lambda^{n} \mathcal{G}$ на $G$, тогда $\mathbf{h}=\left\{X_{1}, \ldots, X_{n}\right\}-$ подалгебра Ли алгебры $\mathcal{G}$.

СлеДСТвИЕ 1. Существует взаимно однозначное соответствие с точностъю до коэфбициента (функиии) между семейством левоинвариантных тензоров Намбу порядка $n$ на $G$ и семейством $n$-мерных подалгебр Ли алгебры $\mathcal{G}$.

Отметим, что если $\eta$ - тензор Намбу порядка $n \geqslant 3$, а $f$ - гладкая функция, то f $\eta$ тоже является тензором Намбу [7].

ТеОРема 2 [6]. Пусть $(G, \eta)$ - т-мерная компактная или полупростая группа Намбу-Ли и элемент $\eta$ имеет высший порядок, тогда $\eta=0$.

Следующая теорема, доказанная в работе [13], предлагает одну из характеристик групп Намбу-Ли.

ТЕОрема 3. Если $G$ - связанная группа Ли, на которой задан тензор Намбу $\eta$, равный нулю в единице е группь $G$, то $(G, \eta)$ является группой Намбу-Ли тогда u только тогда, когда п-скобка любых $n$ левоинвариантных (правоинвариантных) 1-форм группь $G$ является левоинвариантной (правоинвариантной) 1-формой.

Используя эту теорему, можно охарактеризовать мультипликативный тензор $\eta$ высшего порядка. Пусть $\mathcal{G}$ - алгебра Ли группы $G$ с базисом $X_{1}, \ldots, X_{m}$. Ясно, что левоинвариантные векторные поля можно считать базисом алгебры $\mathcal{G}$. Обозначим эти левоинвариантные векторные поля теми же буквами $X_{i}$. Поскольку $\eta$ является тензором высшего порядка, его можно представить как $\eta=f X_{1} \wedge \cdots \wedge X_{m}$ с некоторой функцией $f \in \mathcal{F}(G)$. Используя эти обозначения, приведем теорему из работы [15].

Теорема 4. Пусть $\eta=f X_{1} \wedge \cdots \wedge X_{m}$ - тензор высшего порядка на группе $G$ (такой тензор всегда является тензором Намбу), где $f \in \mathcal{F}(G)$. Тензор $\eta$ является мультипликативным тогда и только тогда, когда $f(e)=0 u$

$$
X_{i} f+\left(\sum_{k=1}^{m} C_{i k}^{k}\right) f=q_{i}, \quad i=1, \ldots, m,
$$

где $C_{i j}^{k}$ - структурные константы алгебры $\mathcal{G}$ по отношению $к$ базису $X_{1}, \ldots, X_{m}$, а $q_{i}$ - некоторые константы.

На основании этой теоремы в работе [15] были получены структуры Намбу третьего порядка для трехмерных действительных групп Ли. В настоящей статье тем же способом вводится структура Намбу четвертого порядка (высшего порядка) для четырехмерных действительных групп Ли, а также с помощью теоремы 1 строится структура Намбу третьего порядка для этих групп Ли. 


\section{3. СТРУКТУРЫ НАМБУ НА ЧЕТЫРЕХМЕРНЫХ ДЕЙСТВИТЕЛЬНЫХ ГРУППАХ ЛИ}

C помощью теорем 1 и 4 в этом разделе мы построим структуры Намбу четвертого и третьего порядков на четырехмерных действительных группах Ли. Отметим, что мы используем классификацию Патеры-Винтерница [17] для четырехмерных алгебр Ли и их подалгебр.

Обозначим через $\mathcal{G}$ четырехмерную действительную алгебру Ли, соответствующую односвязной группе Ли $G$, а также обозначим через $X_{1}, X_{2}, X_{3}, X_{4}$ левоинвариантные векторные поля. Чтобы рассчитать эти векторные поля, нужно определить левоинвариантные 1-формы; такие расчеты были уже проведены в работе [18]. Мы воспользуемся этими результатами для получения левоинвариантных векторных полей. В общем случае для группы Ли $G$ с алгеброй Ли $\mathcal{G}$ и абстрактного базиса $\left\{T_{i}\right\}$ левоинвариантные 1-формы можно ввести следующим образом:

$$
g^{-1} d g=e_{\mu}^{i} T_{i} d x^{\mu}, \quad g \in G,
$$

при этом для левоинвариантных векторных полей получим выражения [18]

$$
X_{i}=V_{i}^{\mu} \partial_{\mu},
$$

где $V_{i}{ }^{\mu}=\left(e_{i}{ }^{\mu}\right)^{\mathrm{t}}, e_{i}{ }^{\mu}$ - тензор, обратный к $e_{\mu}^{i}$, а верхний индекс $\mathrm{t}$ означает транспонирование. Таким образом можно найти левоинвариантные векторные поля. Результаты представлены в табл. 1 в приложении.

Теперь, чтобы рассчитать структуру Намбу $\eta \in \Gamma\left(\Lambda^{4} T G\right)$, представим ее в виде $\eta=f X_{1} \wedge X_{2} \wedge X_{3} \wedge X_{4}$, а для расчета структуры Намбу $\eta \in \Gamma\left(\Lambda^{3} T G\right)$ ее можно представить в виде $\eta=f X_{1} \wedge X_{2} \wedge X_{3}$, где $f \in \mathcal{F}(G)$. Затем определим структуры Намбу четвертого порядка на четырехмерных действительных группах Ли, используя теорему 4, а с помощью теорем 1 и 4 найдем структуры Намбу третьего порядка на действительных четырехмерных группах Ли.

Продемонстрируем, как работает наш метод на примере алгебры Ли $A_{4,8}$. Эта алгебра Ли изоморфна алгебре Гейзенберга, и для нее справедливы следующие коммутационные соотношения [17]:

$$
\left[X_{2}, X_{4}\right]=X_{2}, \quad\left[X_{3}, X_{4}\right]=-X_{3}, \quad\left[X_{2}, X_{3}\right]=X_{1} .
$$

Левоинвариантные векторные поля для этой алгебры Ли имеют вид

$$
X_{1}=\frac{\partial}{\partial x^{1}}, \quad X_{2}=\left(-x^{3} e^{-x^{4}}\right) \frac{\partial}{\partial x^{1}}+e^{-x^{4}} \frac{\partial}{\partial x^{2}}, \quad X_{3}=e^{x^{4}} \frac{\partial}{\partial x^{3}}, \quad X_{4}=\frac{\partial}{\partial x^{4}},
$$

причем эти векторные поля удовлетворяют коммутационным соотношениям (1). В соответствии с теоремой 4 функция $f\left(x^{1}, x^{2}, x^{3}, x^{4}\right)$ должна удовлетворять условиям $f(0,0,0,0)=0$ и

$$
X_{i} f+\left(\sum_{k=1}^{m} C_{i k}^{k}\right) f=q_{i} \quad i=1, \ldots, 4,
$$

где $q_{i}$ - некоторые константы. В результате получим решение уравнения (2) в виде $f=q_{4} x^{4}$, следовательно,

$$
\eta=q_{4} x^{4} \frac{\partial}{\partial x^{1}} \wedge \frac{\partial}{\partial x^{2}} \wedge \frac{\partial}{\partial x^{3}} \wedge \frac{\partial}{\partial x^{4}}
$$


что порождает структуру Намбу-Ли четвертого порядка на соответствующей группе Ли $\mathbf{A}_{4,8}$.

Таким же образом получим следующие левоинвариантные векторные поля для трехмерных подалгебр Ли алгебры $A_{4,8}[17]$ :

для подалгебры $A_{3,1}$ набор левоинвариантных векторных полей $\left\{X_{2}, X_{3} ; X_{1}\right\}$ задается как

$$
X_{2}=\frac{\partial}{\partial x^{2}}-x_{3} \frac{\partial}{\partial x^{1}}, \quad X_{3}=\frac{\partial}{\partial x^{3}} ; \quad X_{1}=\frac{\partial}{\partial x^{1}} ;
$$

для подалгебры $A_{2} \oplus A_{1}$ мы имеем набор $\left\{X_{4}, X_{1} ; X_{2}\right\}$, где

$$
X_{4}=\frac{\partial}{\partial x^{4}}+x_{2} \frac{\partial}{\partial x^{2}}, \quad X_{1}=\frac{\partial}{\partial x^{1}} ; \quad X_{2}=\frac{\partial}{\partial x^{2}}
$$

для подалгебры $A_{2} \oplus A_{1}$ набор левоинвариантных векторных полей $\left\{X_{4}, X_{1} ; X_{3}\right\}$ задается как

$$
X_{4}=\frac{\partial}{\partial x^{4}}-x_{3} \frac{\partial}{\partial x^{3}}, \quad X_{1}=\frac{\partial}{\partial x^{1}} ; \quad X_{3}=\frac{\partial}{\partial x^{3}} .
$$

Это порождает следующие структуры Намбу третьего порядка на группе $\mathbf{A}_{4,8}$ (соответственно):

$$
\begin{aligned}
& \eta_{1}=\left(q_{1} x^{2}+q_{2} x^{3}\right) \frac{\partial}{\partial x^{1}} \wedge \frac{\partial}{\partial x^{2}} \wedge \frac{\partial}{\partial x^{3}} \\
& \eta_{2}=\left(q_{3} x^{2}+q_{1}\left(e^{x^{4}}-1\right)\right) \frac{\partial}{\partial x^{1}} \wedge \frac{\partial}{\partial x^{2}} \wedge \frac{\partial}{\partial x^{4}} \\
& \eta_{3}=\left(q_{3} x^{3}+q_{1}\left(e^{-x^{4}}-1\right)\right) \frac{\partial}{\partial x^{1}} \wedge \frac{\partial}{\partial x^{3}} \wedge \frac{\partial}{\partial x^{4}} .
\end{aligned}
$$

Таким образом, мы задали все структуры Намбу четвертого и третьего порядков на четырехмерных действительных группах Ли.

Список результатов приведен в табл. 1 и 2 приложения.

\section{4. СУПЕРИНТЕГРИРУЕМЫЕ СИСТЕМЫ СО СТРУКТУРАМИ НАМБУ}

Гамильтонова система с $n$ степенями свободы является интегрируемой в смысле Лиувилля, если у нее есть $n$ инвариантов, находящихся в инволюции [19], и является суперинтегрируемой, если у нее есть дополнительно вплоть до $2 n-1$ независимых инвариантов [20]. В этом разделе мы построим суперинтегрируемые динамические системы с помощью структур Намбу четвертого порядка на четырехмерных действительных группах Ли. Фактически мы рассмотрим некоторые системы, связанные со структурой Намбу на некоторых группах Ли, выступающих в качестве фазового пространства (т. е. группах Ли, наделенных симплектической структурой [16]). Эту работу мы выполним в два этапа. На первом этапе рассмотрим суперинтегрируемые системы с $\mathbf{A}_{4,8}$ в качестве группы симметрий. На втором этапе рассмотрим системы с $\mathbf{A}_{4,10}$ в качестве группы симметрий. Заметим, что $\mathbf{A}_{4,8}$ и $\mathbf{A}_{4,10}$ - единственные группы, имеющие обратимые ad-инвариантные метрики. В дальнейшем $\left(x^{1}, \ldots, x^{4}\right)-$ координаты групп Ли. 


\section{1. Суперинтегрируемые системы с группой $\mathbf{A}_{4,8}$ в качестве группы} симметрий. Рассмотрим несколько случаев.

- Группа Ли $\mathbf{A}_{4,9}^{0}$ в качестве фазового пространства. Симплектическая структура на группе $\mathbf{A}_{4,9}^{0}$ имеет вид [16]

$$
\left\{x^{1}, x^{4}\right\}=\alpha, \quad\left\{x^{2}, x^{3}\right\}=-\alpha,
$$

где $\alpha$ - произвольная ненулевая действительная константа. Введем координаты Дарбу

$$
y^{1}=-\frac{1}{\alpha} x^{2}, \quad y^{2}=x^{1}, \quad y^{3}=x^{3}, \quad y^{4}=\frac{1}{\alpha} x^{4},
$$

которые удовлетворяют стандартным скобкам Пуассона ${ }^{1)}$

$$
\left\{y^{1}, y^{3}\right\}=1, \quad\left\{y^{2}, y^{4}\right\}=1 .
$$

Теперь, используя упомянутый в работе [21] метод, мы можем построить динамическую систему с помощью структуры Намбу на группе Ли $\mathbf{A}_{4,9}^{0}$ в качестве фазового пространства. Для этой цели рассмотрим динамические величины $Q_{a}$ как функции переменных $x^{i}$, которые удовлетворяют следующим соотношениям:

$$
\left\{Q_{a}, Q_{b}\right\}=f_{a b}^{c} Q_{c},
$$

где $f_{a b}^{c}$ - структурная константа алгебры симметрий Ли $A_{4,8}$. Далее, проведя некоторые расчеты, выразим 4-скобки Намбу (с весами, равными структурным константам) через скобку Пуассона [21]:

$$
g^{a c} g^{b d} f_{c d}^{e}\left\{A, Q_{a}, Q_{b}, Q_{e}\right\}=3 g^{a c} g^{b d} f_{c d}^{e} \eta\left\{A, Q_{a}\right\}\left\{Q_{b}, Q_{e}\right\}=3 g^{a c} g^{b d} f_{c d}^{e} f_{b e}^{f} \eta\left\{A, Q_{a}\right\} Q_{f},
$$

где $g^{a c}$ - метрика, обратная к ad-инвариантной невырожденной метрике на алгебре Ли $A_{4,8}$, а $\eta$ - структура Намбу на группе Ли $\mathbf{A}_{4,9}^{0}$, выраженная через координаты $y^{i}$, $i=1,2,3,4$. После некоторых вычислений получим, что ad-инвариантная метрика для алгебры Ли $A_{4,8}$ имеет следующий вид:

$$
g_{a b}=\left(\begin{array}{rrrr}
0 & 0 & 0 & s \\
0 & 0 & -s & 0 \\
0 & -s & 0 & 0 \\
s & 0 & 0 & 0
\end{array}\right)
$$

где $s$ - произвольная ненулевая действительная постоянная.

С другой стороны, после некоторых вычислений получим выражение

$$
g^{a c} g^{b d} f_{c d}^{e} f_{b e}^{f} Q_{a} Q_{f}=-\frac{2}{s^{2}} Q_{1}^{2},
$$

где $Q_{1}$ - функция Казимира алгебры $A_{4,8}[22]$; в то же время для динамической системы с алгеброй симметрий Ли $A_{4,8}$ это выражение пропорционально гамильтониану системы. В связи с этим имеем

$$
g^{a c} g^{b d} f_{c d}^{e}\left\{A, Q_{a}, Q_{b}, Q_{e}\right\}=-\frac{3 \eta}{s^{2}}\{A, H\}=-\frac{3 \eta}{s^{2}} \frac{\partial A}{\partial t},
$$

1) Отметим, что в последующих примерах все координаты Дарбу удовлетворяют стандартным скобкам Пуассона (4). 
где $\eta$ - значение структуры Намбу группы Ли $\mathbf{A}_{4,9}^{0}$ и $H=Q_{1}^{2}$. Поэтому эволюцию динамической системы можно описать с помощью структуры Намбу следующим образом:

$$
\frac{\partial A}{\partial t}=-\frac{s^{2}}{3 \eta} g^{a c} g^{b d} f_{c d}^{e}\left\{A, Q_{a}, Q_{b}, Q_{e}\right\} .
$$

Теперь, используя реализацию алгебры Ли $A_{4,8}[23]$ в $\mathbb{R}^{4}$ вида

$$
X_{1}=\frac{\partial}{\partial y_{1}}, \quad X_{2}=\frac{\partial}{\partial y_{2}}, \quad X_{3}=y^{2} \frac{\partial}{\partial y_{1}}, \quad X_{4}=y^{2} \frac{\partial}{\partial y_{2}},
$$

получим следующие выражения ${ }^{2)}$ для $Q_{i}$ :

$Q_{1}=-p_{1}=-y^{3}, \quad Q_{2}=-p_{2}=-y^{4}, \quad Q_{3}=-y^{2} p_{1}=-y^{2} y^{3}, \quad Q_{4}=-y^{2} p_{2}=-y^{2} y^{4}$.

Они удовлетворяют условию (5) в силу соотношений (4). Соответствующий гамильтониан можно представить следующим образом:

$$
H=p_{1}^{2}=\left(y^{3}\right)^{2} .
$$

Таким образом, динамику суперинтегрируемой системы (с инволютивными функциями ${ }^{3)}$, скажем, $\left.\left(H, Q_{1}, Q_{2}\right)\right)$ можно описать с помощью структур Намбу ${ }^{4)}$ :

$$
\frac{\partial A}{\partial t}=-\frac{s^{2}}{3 q_{4}\left(e^{-2 \alpha y^{4}}-1\right)} g^{a c} g^{b d} f_{c d}^{e}\left\{A, Q_{a}, Q_{b}, Q_{e}\right\},
$$

где использовалось равенство $\eta=q_{4}\left(e^{-2 x^{4}}-1\right)$ (см. табл. 1) и координаты Дарбу (3).

- Группа Ли $\mathbf{A}_{4,2}^{-1}$ в качестве фазового пространства. Для этой группы Ли симплектическая структура имеет следующий вид [16]:

$$
\left\{x^{1}, x^{2}\right\}=2 \alpha, \quad\left\{x^{1}, x^{3}\right\}=-\alpha, \quad\left\{x^{2}, x^{4}\right\}=\beta e^{-x^{4}},
$$

где $\alpha$ и $\beta$ - произвольные ненулевые действительные числа. Координаты Дарбу для группы $\mathbf{A}_{4,2}^{-1}$ суть [24]

$$
y^{1}=-\frac{e^{x^{4}}}{\beta}+x^{3}, \quad y^{2}=-\frac{2 \alpha e^{x^{4}}-\beta x^{1}+\alpha \beta x^{2}}{\alpha \beta^{2}}, \quad y^{3}=\frac{2 e^{x^{4}}}{\beta}+\frac{x^{1}}{\alpha}, \quad y^{4}=e^{x^{4}},
$$

при этом структура Намбу на групе Ли $\mathbf{A}_{4,2}^{-1}$ (см. табл. 1) выражается через координаты $y^{i}, i=1,2,3,4$, следующим образом: $\eta=q_{4}\left(1 / y^{4}-1\right)$. Поэтому динамику суперинтегрируемой системы можно описать с помощью уравнения (8):

$$
\frac{\partial A}{\partial t}=-\frac{s^{2}}{3 q_{4}\left(1 / y^{4}-1\right)} g^{a c} g^{b d} f_{c d}^{e}\left\{A, Q_{a}, Q_{b}, Q_{e}\right\} .
$$

При этом гамильтониан имеет вид

$$
H=\left(y^{3}\right)^{2}=\left(\frac{2 e^{x^{4}}}{\beta}+\frac{x^{1}}{\alpha}\right)^{2} .
$$

\footnotetext{
2) Отметим, что мы используем квантово-механическую реализацию операторов $p_{i}=-\partial / \partial y_{i}$, при этом $p_{1}=y^{3}, p_{2}=y^{4}$ для наших стандартных скобок Пуассона (4).

3) В последующих примерах инволютивные функции можно задать одним из следующих наборов: $\left(H, Q_{1}, Q_{2}\right),\left(H, Q_{1}, Q_{3}\right)$ или $\left(H, Q_{1}, Q_{4}\right)$.

4) Отметим, что все полученные далее системы имеют ту же динамическую эволюцию, что и эволюция, заданная уравнением (8), и тот же гамильтониан (9) (в координатах Дарбу); отличаются только значения $\eta$ (коэффициентная функция структуры Намбу).
} 
- Группа Ли $\mathbf{A}_{4,3}$ в качестве фазового пространства. Невырожденная структура Пуассона на $\mathbf{A}_{4,3}$ имеет вид [16]

$$
\left\{x^{1}, x^{2}\right\}=\alpha x^{4} e^{-x^{4}}, \quad\left\{x^{1}, x^{3}\right\}=\beta e^{-x^{4}}, \quad\left\{x^{1}, x^{4}\right\}=\gamma e^{-x^{4}}, \quad\left\{x^{2}, x^{3}\right\}=\lambda,
$$

где $\alpha, \beta, \gamma$ и $\lambda$ - произвольные ненулевые действительные числа. Координаты Дарбу для группы $\mathbf{A}_{4,3}$ записываются как [24]

$$
\begin{gathered}
y^{1}=\frac{\beta x^{2}}{\lambda}+\frac{\alpha \gamma\left(x^{3}\right)^{2}}{2 \beta \lambda}-\frac{\alpha x^{3} x^{4}}{\lambda}, \quad y^{2}=\frac{x^{1}}{\gamma}-\frac{\beta e^{-x^{4}} x^{2}}{\gamma \lambda}-\frac{\alpha e^{-x^{4}}\left(x^{3}\right)^{2}}{2 \beta \lambda}+\frac{\alpha e^{-x^{4}} x^{3} x^{4}}{\gamma \lambda}, \\
y^{3}=\frac{x^{3}}{\beta}, \quad y^{4}=e^{x^{4}}
\end{gathered}
$$

таким образом, структура Намбу на группе Ли $\mathbf{A}_{4,3}$ (см. табл. 1) выражается через координаты $y^{i}, i=1,2,3,4$, следующим образом:

$$
\eta=q_{1} \gamma y^{2}+q_{1} \frac{y^{1}}{y^{4}}+\frac{\alpha q_{1} y^{3} \ln y^{4}}{2 \lambda y^{4}}-\frac{q_{1} \alpha \beta y^{3} \ln y^{4}}{\lambda y^{4}}+\frac{q_{4}}{y^{4}}-q_{4} .
$$

Динамику суперинтегрируемой системы можно описать, используя уравнение (8) и скобку Намбу:

$$
\frac{\partial A}{\partial t}=-\frac{s^{2}}{3\left(q_{1} \gamma y^{2}+q_{1} \frac{y^{1}}{y^{4}}+\frac{\alpha q_{1} y^{3} \ln y^{4}}{2 \lambda y^{4}}-\frac{q_{1} \alpha \beta y^{3} \ln y^{4}}{\lambda y^{4}}+\frac{q_{4}}{y^{4}}-q_{4}\right)} g^{a c} g^{b d} f_{c d}^{e}\left\{A, Q_{a}, Q_{b}, Q_{e}\right\}
$$

с гамильтонианом

$$
H=\left(y^{3}\right)^{2}=\left(\frac{x^{3}}{\beta}\right)^{2}
$$

- Группа Ли $\mathbf{A}_{4,6}^{a, 0}$ в качестве фазового пространства. Для этой группы Ли невырожденная структура Пуассона имеет вид [16]

$$
\left\{x^{1}, x^{4}\right\}=\gamma e^{-\alpha x^{4}}, \quad\left\{x^{2}, x^{3}\right\}=\beta,
$$

где $\alpha, \beta$ и $\gamma-$ произвольные ненулевые действительные числа. Координаты Дарбу для группы Ли $\mathbf{A}_{4,6}^{a, 0}$ суть [24]

$$
y^{1}=x^{3}, \quad y^{2}=\frac{e^{2 \alpha x^{4}} x^{1}}{\alpha \gamma}, \quad y^{3}=-\frac{x^{2}}{\beta}, \quad y^{4}=e^{-\alpha x^{4}},
$$

поэтому структуру Намбу на группе Ли $\mathbf{A}_{4,6}^{a, 0}$ (см. табл. 1) можно выразить через координаты $y^{i}, i=1,2,3,4$, как

$$
\eta=q_{1} \alpha \gamma\left(y^{4}\right)^{2} y^{2}+q_{4} y^{4}-q_{4},
$$

а динамика суперинтегрируемой системы описывается с помощью уравнения (8) следующим образом:

$$
\frac{\partial A}{\partial t}=-\frac{s^{2}}{3\left(q_{1} \alpha \gamma\left(y^{4}\right)^{2} y^{2}+q_{4} y^{4}-q_{4}\right)} g^{a c} g^{b d} f_{c d}^{e}\left\{A, Q_{a}, Q_{b}, Q_{e}\right\},
$$

при этом гамильтониан имеет вид

$$
H=\left(y^{3}\right)^{2}=\left(\frac{x^{2}}{\beta}\right)^{2} .
$$


- Группа Ли $\mathbf{A}_{4,7}$ в качестве фазового пространства. Невырожденная структура Пуассона на группе $\mathbf{A}_{4,7}$ имеет вид [16]

$$
\left\{x^{1}, x^{3}\right\}=-2 \alpha x^{3} e^{-2 x^{4}}, \quad\left\{x^{1}, x^{4}\right\}=\alpha e^{-2 x^{4}}, \quad\left\{x^{2}, x^{3}\right\}=2 \alpha e^{-2 x^{4}},
$$

где $\alpha$ - произвольное ненулевое действительное число. Координаты Дарбу для $\mathbf{A}_{4,7}$ выглядят следующим образом [24]:

$$
y^{1}=\frac{e^{2 x^{4}} x^{2}}{2 \alpha}, \quad y^{2}=-\frac{-1-e^{2 x^{4}}+e^{4 x^{4} x^{1}+e^{4 x^{4}} x^{2} x^{3}}}{2 \alpha}, \quad y^{3}=x^{3}, \quad y^{4}=e^{-2 x^{4}},
$$

следовательно, структуру Намбу на группе Ли $\mathbf{A}_{4,7}$ (см. табл. 1) можно выразить через координаты $y^{i}, i=1,2,3,4$, как

$$
\eta=q_{4}\left(\left(y^{4}\right)^{2}-1\right)
$$

Таким образом, динамические уравнения суперинтегрируемой системы можно получить из уравнения (8), мы имеем

$$
\frac{\partial A}{\partial t}=-\frac{s^{2}}{3 q_{4}\left(\left(y^{4}\right)^{2}-1\right)} g^{a c} g^{b d} f_{c d}^{e}\left\{A, Q_{a}, Q_{b}, Q_{e}\right\},
$$

где гамильтониан имеет вид

$$
H=\left(y^{3}\right)^{2}=\left(x^{3}\right)^{2} .
$$

- Группа Ли $\mathbf{A}_{4,9}^{1}$ в качестве фазового пространства. Симплектическая структура на группе $\mathbf{A}_{4,9}^{1}$ выглядит следующим образом [16]:

$$
\left\{x^{1}, x^{3}\right\}=2 \alpha x^{3} e^{-2 x^{4}}, \quad\left\{x^{1}, x^{4}\right\}=-\alpha e^{-2 x^{4}}, \quad\left\{x^{2}, x^{3}\right\}=-2 \alpha e^{-2 x^{4}},
$$

где $\alpha$ - произвольное ненулевое действительное число. Координаты Дарбу для группы Ли $\mathbf{A}_{4,9}^{1}$ имеют вид [24]

$$
y^{1}=-\frac{e^{2 x^{4}} x^{2}}{2 \alpha}, \quad y^{2}=\frac{-1-e^{2 x^{4}}+e^{4 x^{4}} x^{1}+e^{2 x^{4}} x^{2} x^{3}}{2 \alpha}, \quad y^{3}=x^{3}, \quad y^{4}=e^{-2 x^{4}},
$$

таким образом, структуру Намбу на группе Ли $\mathbf{A}_{4,9}^{1}$ (см. табл. 1) можно выразить через координаты $y^{i}, i=1,2,3,4$, как и выше, с помощью формулы (10). При этом динамическое уравнение суперинтегрируемой системы принимает вид (11) с гамильтонианом (12).

- Группа Ли $\mathbf{A}_{4,12}$ в качестве фазового пространства. Невырожденная структура Пуассона на группе $\mathbf{A}_{4,12}$ выглядит следующим образом [16]:

$$
\begin{array}{ll}
\left\{x^{1}, x^{3}\right\}=-\gamma e^{-x^{3}}\left(\alpha \cos x^{4}+\beta \sin x^{4}\right), \quad\left\{x^{1}, x^{4}\right\}=\gamma e^{-x^{3}}\left(-\beta \cos x^{4}+\alpha \sin x^{4}\right), \\
\left\{x^{2}, x^{3}\right\}=\gamma e^{-x^{3}}\left(-\beta \cos x^{4}-\alpha \sin x^{4}\right), \quad\left\{x^{2}, x^{4}\right\}=-\gamma e^{-x^{3}}\left(\alpha \cos x^{4}+\beta \sin x^{4}\right),
\end{array}
$$

где $\alpha$ и $\beta$ - произвольные ненулевые действительные числа, $\gamma=1 /\left(\alpha^{2}+\beta^{2}\right)$. Координаты Дарбу на группе $\mathbf{A}_{4,12}$ введем следующим образом [24]:

$$
\begin{aligned}
& y^{1}=e^{2 x^{3}}\left(\alpha x^{1} \cos x^{4}-\beta x^{2} \cos x^{4}+\beta x^{1} \sin x^{4}+\alpha x^{2} \sin x^{4}\right), \\
& y^{2}=e^{-x^{3}}\left(\beta x^{1} \cos x^{4}+\alpha x^{2} \cos x^{4}-\alpha x^{1} \sin x^{4}+\beta x^{2} \sin x^{4}\right), \\
& y^{3}=e^{x^{3}}, \quad y^{4}=x^{4},
\end{aligned}
$$


тогда структура Намбу на группе Ли $\mathbf{A}_{4,12}$ (см. табл. 1) выражается через координаты $y^{i}, i=1,2,3,4$, как

$$
\eta=q_{4}\left(\frac{1}{\left(y^{3}\right)^{2}}-1\right)
$$

при этом динамическое уравнение суперинтегрируемой системы получается из уравнения (8) в виде

$$
\frac{\partial A}{\partial t}=-\frac{s^{2}}{3 q_{4}\left(1 /\left(y^{3}\right)^{2}-1\right)} g^{a c} g^{b d} f_{c d}^{e}\left\{A, Q_{a}, Q_{b}, Q_{e}\right\}
$$

с гамильтонианом

$$
H=\left(y^{3}\right)^{2}=e^{2 x^{3}} .
$$

\section{2. Суперинтегрируемые системы с группой $\mathbf{A}_{4,10}$ в качестве группы} симметрии. Как и в предыдущем пункте, построим некоторые динамические системы с помощью структуры Намбу на некоторых группах Ли, взятых в качестве фазового пространства с группой симметрий $\mathbf{A}_{4,10}$. Найдем ad-инвариантную невырожденную метрику на алгебре Ли $A_{4,10}$ :

$$
g_{a b}=\left(\begin{array}{cccc}
0 & 0 & 0 & m \\
0 & m & 0 & 0 \\
0 & 0 & m & 0 \\
n & 0 & 0 & 0
\end{array}\right)
$$

где $m$ и $n$ - произвольные ненулевые действительные числа. Для этого случая имеем следующие соотношения вместо (7) и (8):

$$
g^{a c} g^{b d} f_{c d}^{e} f_{b e}^{f} Q_{a} Q_{f}=\frac{2}{m n} Q_{1}^{2}
$$

И

$$
\frac{\partial A}{\partial t}=\frac{m n}{3 \eta} g^{a c} g^{b d} f_{c d}^{e}\left\{A, Q_{a}, Q_{b}, Q_{e}\right\}
$$

Отсюда, используя представление [23] в $\mathbb{R}^{4}$ вида

$$
X_{1}=\frac{\partial}{\partial y_{1}}, \quad X_{2}=\frac{\partial}{\partial y_{2}}, \quad X_{3}=y^{1} \frac{\partial}{\partial y_{1}}+y^{2} \frac{\partial}{\partial y_{2}}, \quad X_{4}=y^{2} \frac{\partial}{\partial y_{1}}-y_{1} \frac{\partial}{\partial y_{2}}
$$

имеем

$$
Q_{1}=-y^{3}, \quad Q_{2}=-y^{4}, \quad Q_{3}=-y^{1} y^{3}-y^{2} y^{4}, \quad Q_{4}=-y^{2} y^{3}+y^{1} y^{4} .
$$

При этом гамильтонианы суперинтегрируемых систем с группой симметрий $\mathbf{A}_{4,10}$ задаются теми же формулами, что и в предыдущем пункте, отличие имеется только в динамических уравнениях (8) (т.е. в метриках, обратных к метрикам (6) и (13) в уравнении (8), а также в структурных константах $f_{a b}^{c}$ алгебр $A_{4,8}$ и $\left.A_{4,10}\right)$.

Таким образом, динамические уравнения для действительных четырехмерных групп Ли, взятых в качестве фазовых пространств, представимы в следующем виде:

- для группы $\mathbf{A}_{4,9}^{0}$ в качестве фазового пространства.

$$
\frac{\partial A}{\partial t}=\frac{m n}{3 q_{4}\left(e^{-2 \alpha y^{4}}-1\right)} g^{a c} g^{b d} f_{c d}^{e}\left\{A, Q_{a}, Q_{b}, Q_{e}\right\}
$$


- для группы $\mathbf{A}_{4,2}^{-1}$ в качестве фазового пространства.

$$
\frac{\partial A}{\partial t}=\frac{m n}{3 q_{4}\left(1 / y^{4}-1\right)} g^{a c} g^{b d} f_{c d}^{e}\left\{A, Q_{a}, Q_{b}, Q_{e}\right\},
$$

- для группы $\mathbf{A}_{4,3}$ в качестве фазового пространства.

$$
\begin{aligned}
\frac{\partial A}{\partial t}= & \frac{m n}{3\left(q_{1} \gamma y^{2}+\frac{q_{1} y^{1}}{y^{4}}+\frac{\alpha q_{1} y^{3} \ln y^{4}}{2 \lambda y^{4}}-\frac{q_{1} \alpha \beta y^{3} \ln y^{4}}{\lambda y^{4}}+\frac{q_{4}}{y^{4}}-q_{4}\right)} \times \\
& \times g^{a c} g^{b d} f_{c d}^{e}\left\{A, Q_{a}, Q_{b}, Q_{e}\right\},
\end{aligned}
$$

- для группы $\mathbf{A}_{4,6}^{a, 0}$ в качестве фазового пространства.

$$
\frac{\partial A}{\partial t}=\frac{m n}{3\left(q_{1} \alpha \gamma\left(y^{4}\right)^{2} y^{2}+q_{4} y^{4}-q_{4}\right)} g^{a c} g^{b d} f_{c d}^{e}\left\{A, Q_{a}, Q_{b}, Q_{e}\right\},
$$

- для группы $\mathbf{A}_{4,7}$ в качестве фазового пространства.

$$
\frac{\partial A}{\partial t}=\frac{m n}{3 q_{4}\left(\left(y^{4}\right)^{2}-1\right)} g^{a c} g^{b d} f_{c d}^{e}\left\{A, Q_{a}, Q_{b}, Q_{e}\right\}
$$

- для группы $\mathbf{A}_{4,9}^{1}$ в качестве фазового пространства.

$$
\frac{\partial A}{\partial t}=\frac{m n}{3 q_{4}\left(\left(y^{4}\right)^{2}-1\right)} g^{a c} g^{b d} f_{c d}^{e}\left\{A, Q_{a}, Q_{b}, Q_{e}\right\}
$$

- для группы $\mathbf{A}_{4,12}$ в качестве фазового пространства.

$$
\frac{\partial A}{\partial t}=\frac{m n}{3 q_{4}\left(1 /\left(y^{3}\right)^{2}-1\right)} g^{a c} g^{b d} f_{c d}^{e}\left\{A, Q_{a}, Q_{b}, Q_{e}\right\} .
$$

\section{5. ВЫВОДЫ}

Получены все структуры Намбу четвертого и третьего порядков на четырехмерных действительных группах Ли. Получены также новые суперинтегрируемые системы с некоторыми четырехмерными действительными группами Ли в качестве фазовых пространств и группами симметрий $\mathbf{A}_{4,8}$ и $\mathbf{A}_{4,10}$, при этом уравнения, описывающие их динамику, выражаются через соответствующие структуры Намбу четвертого порядка.

\section{ПРИЛОЖЕНИЕ}

В табл. 1 приведены структуры Намбу четвертого порядка на четырехмерных действительных группах Ли и соответствующие левоинвариантные векторные поля, а в табл. 2 - соответствующие алгебры Ли с левоинвариантными векторными полями и структуры Намбу третьего порядка на группах Ли. В таблицах использовано обозначение $\partial_{i}=\partial / \partial x_{i}, i=1,2,3,4$. 


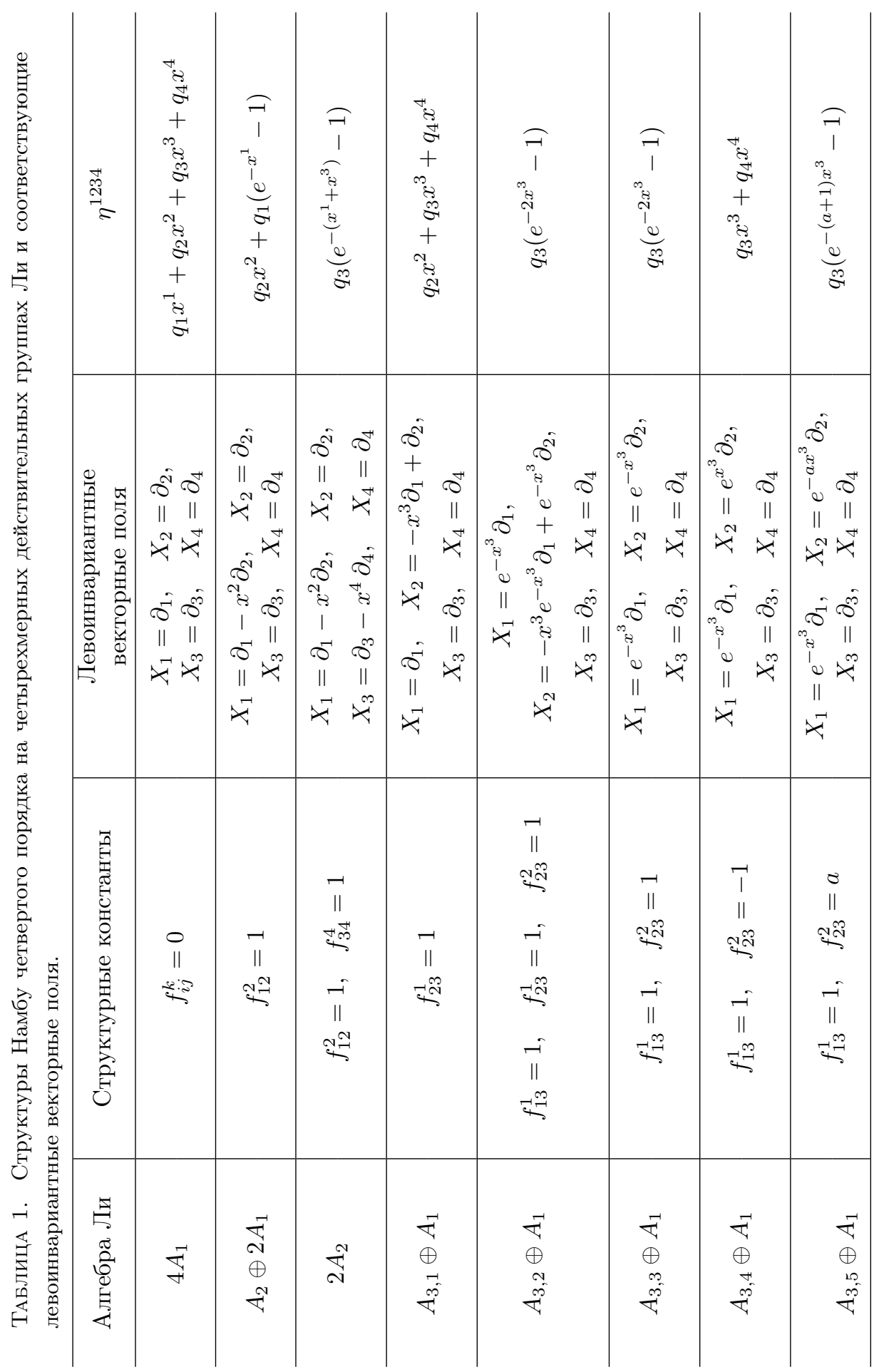




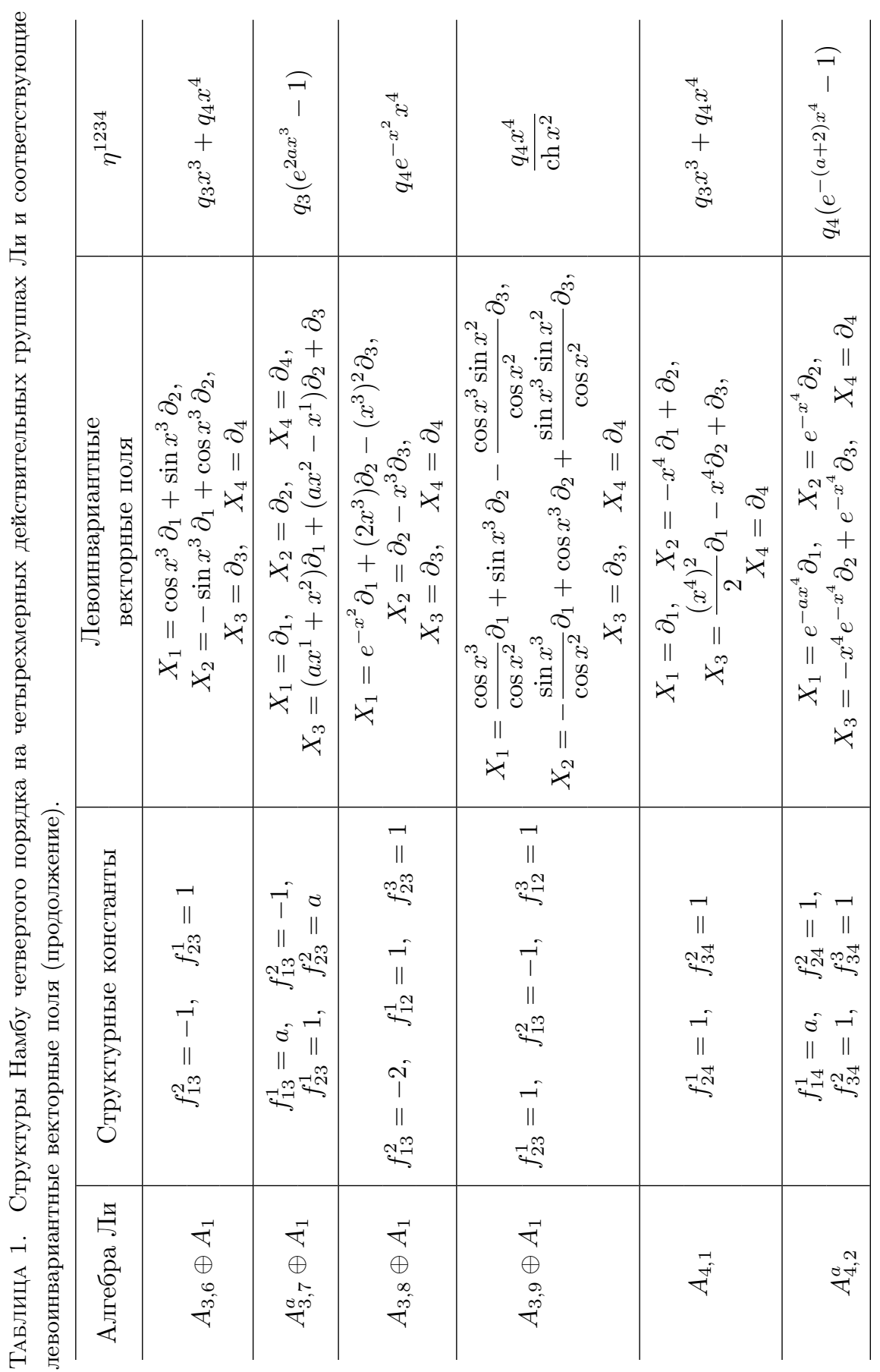




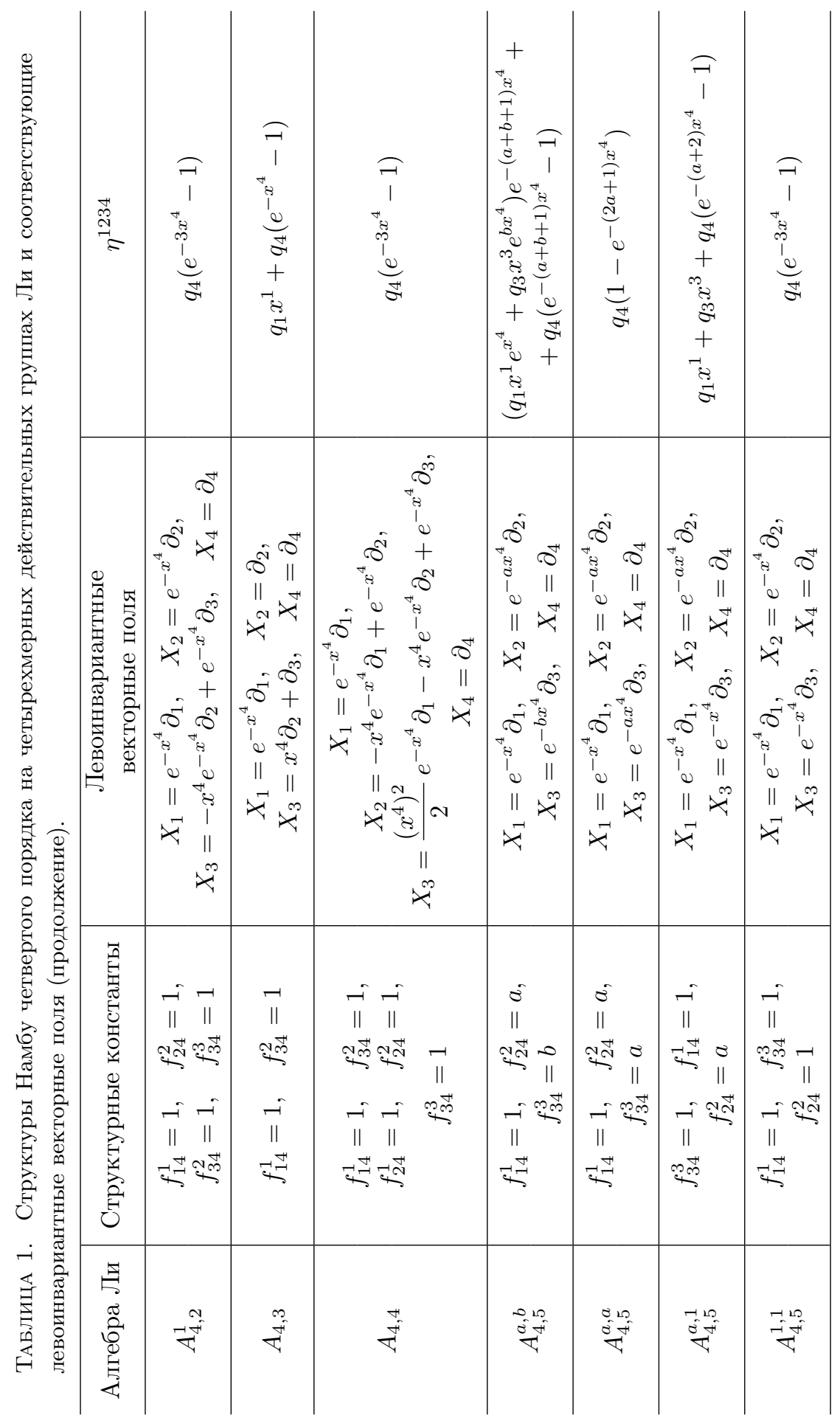




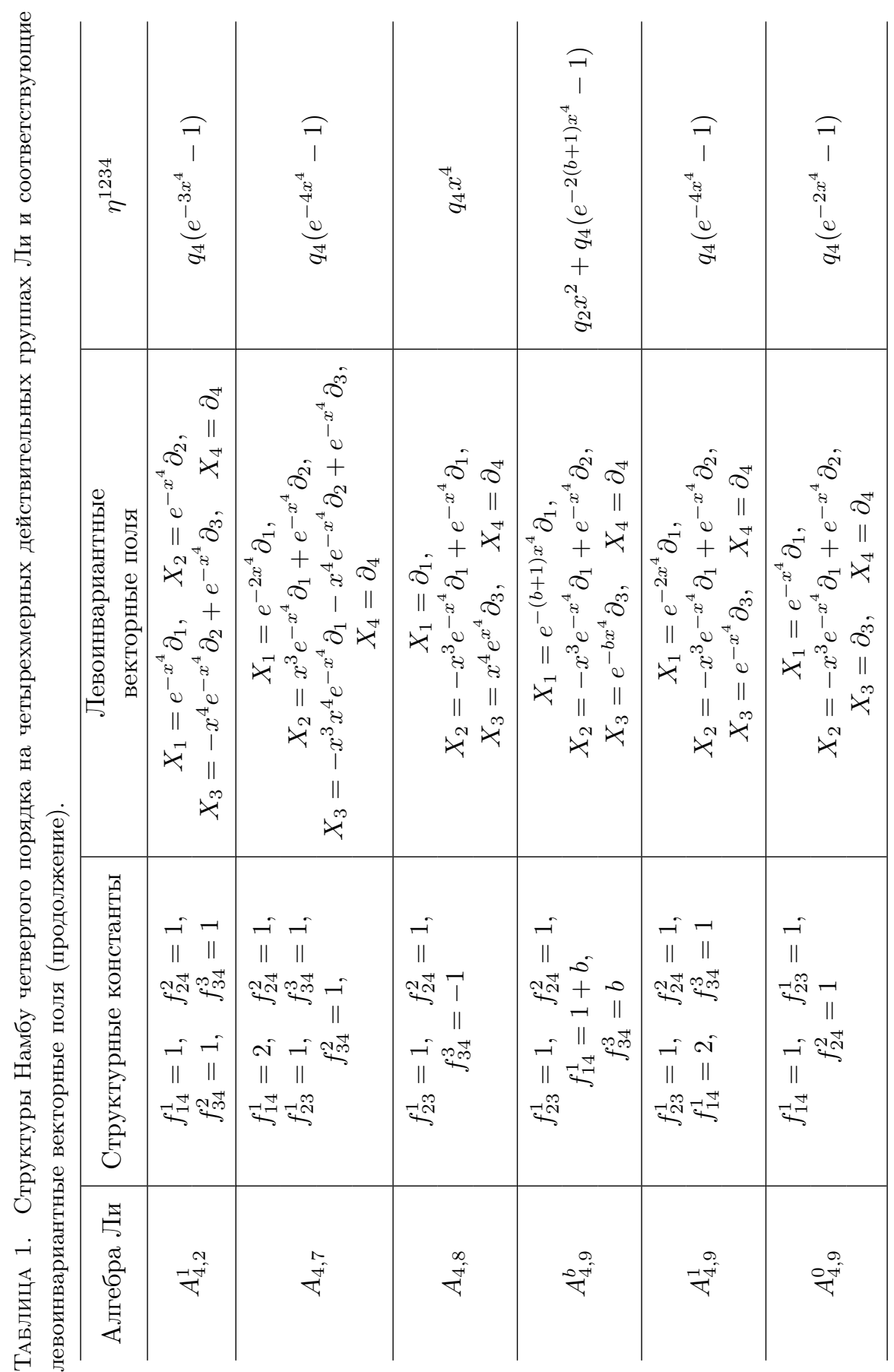




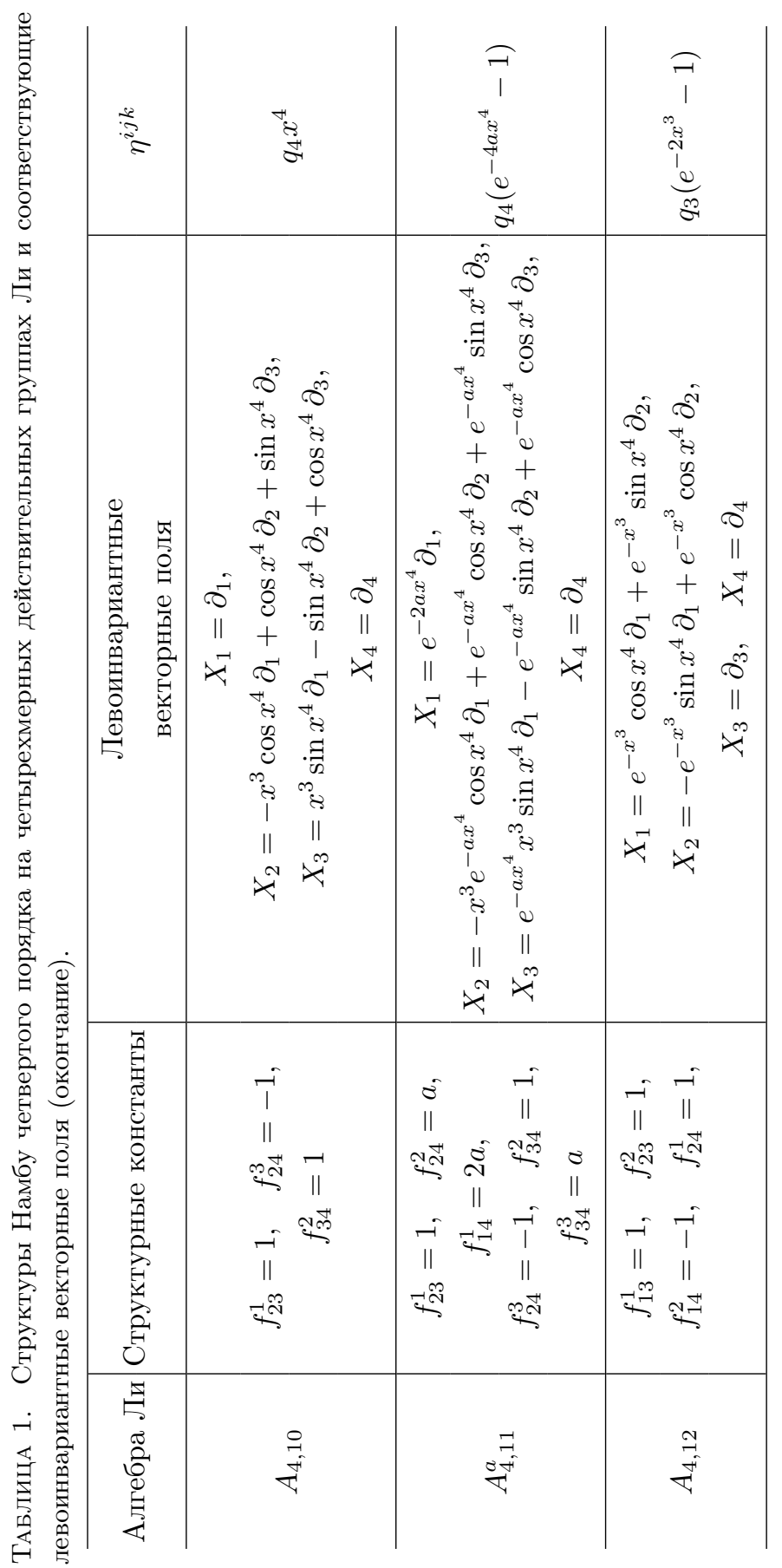




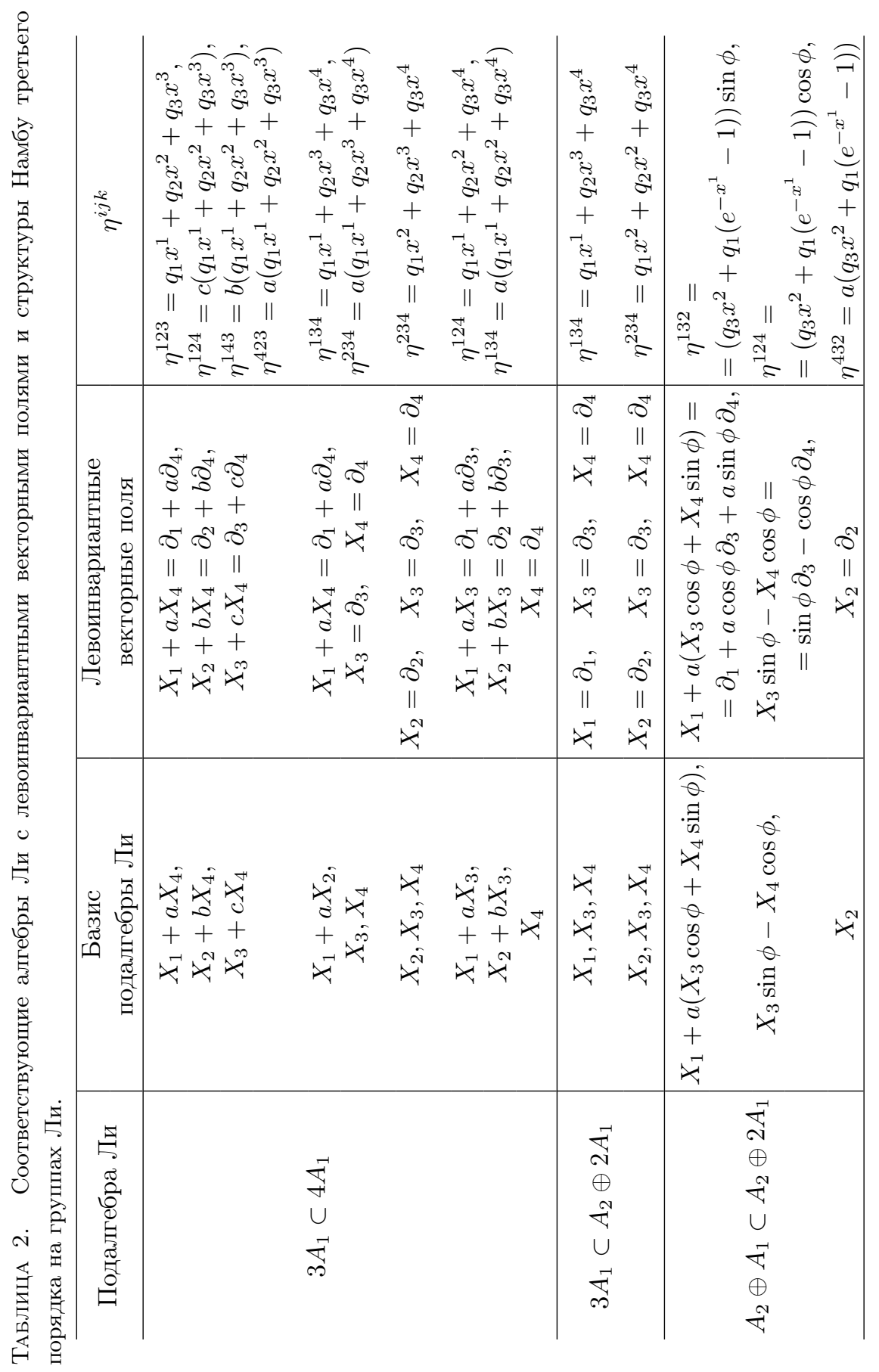




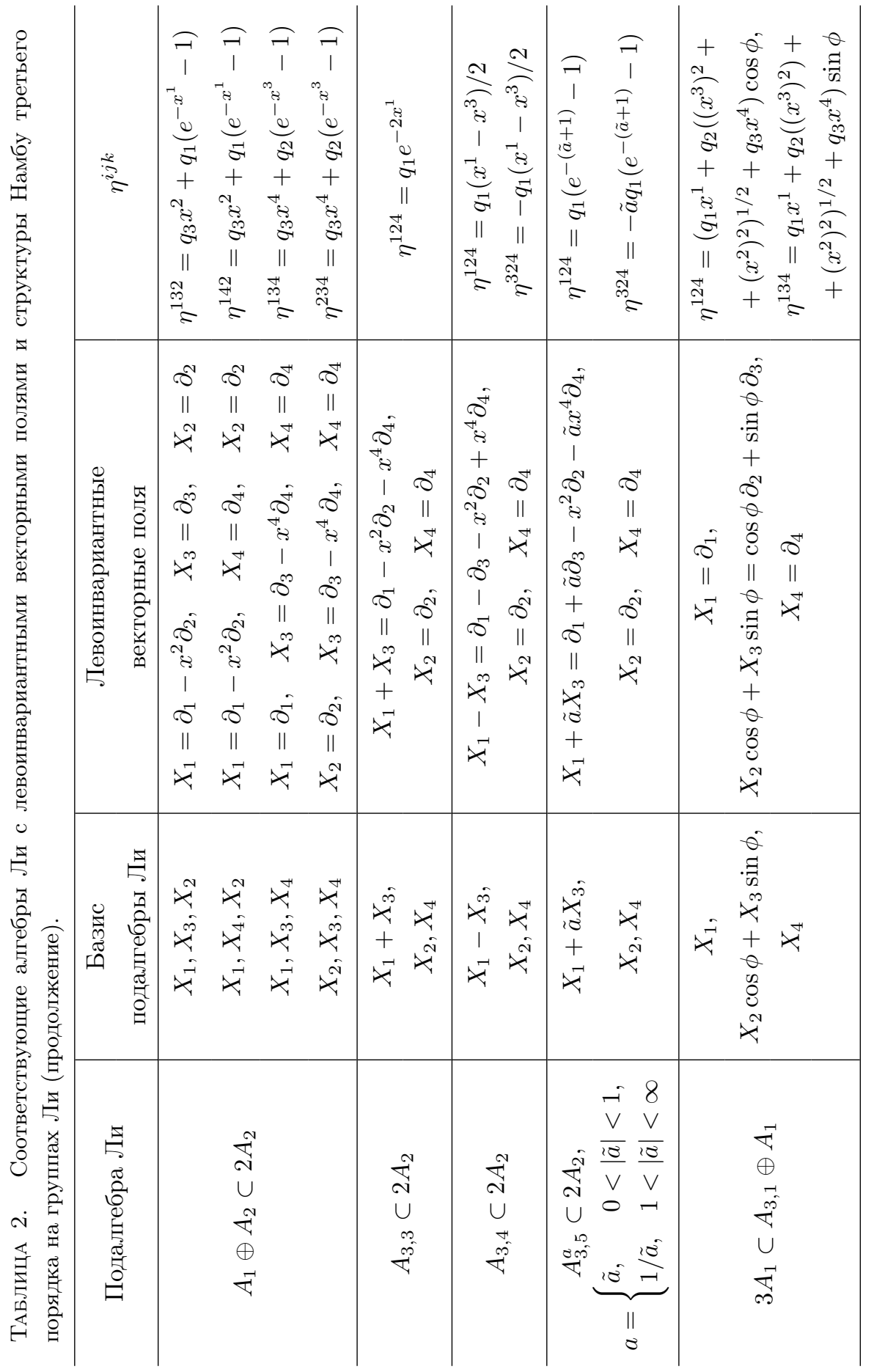




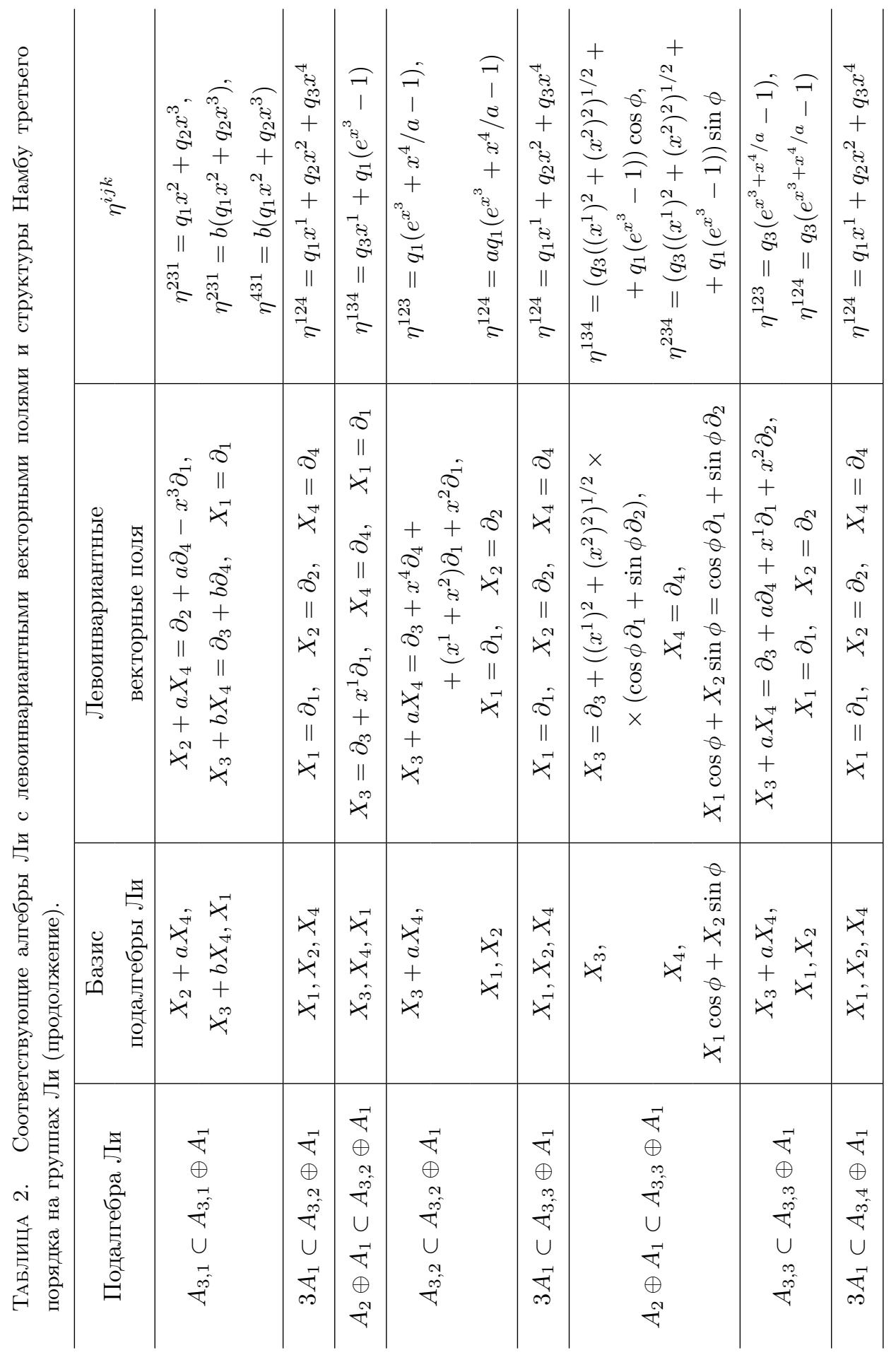




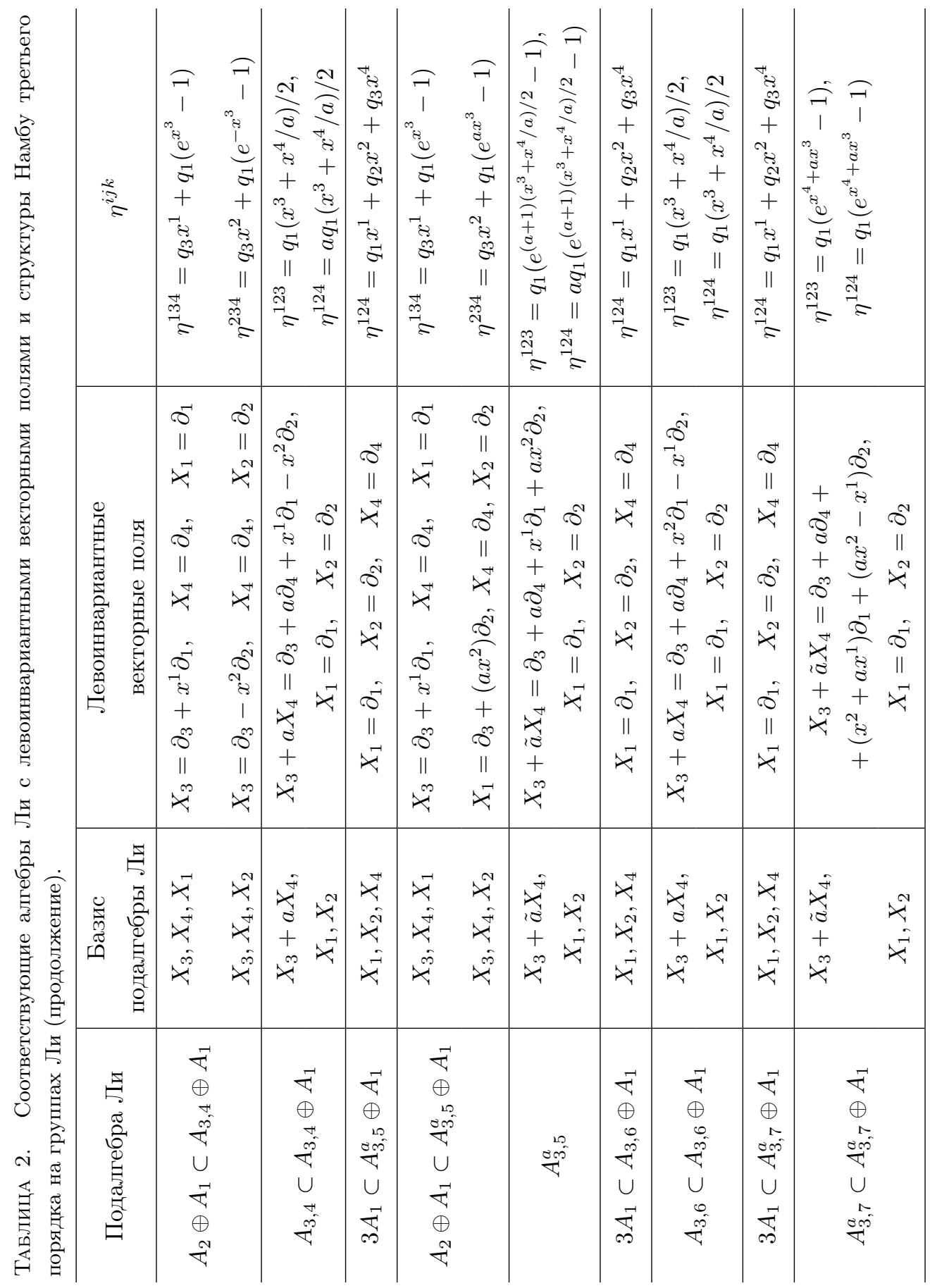




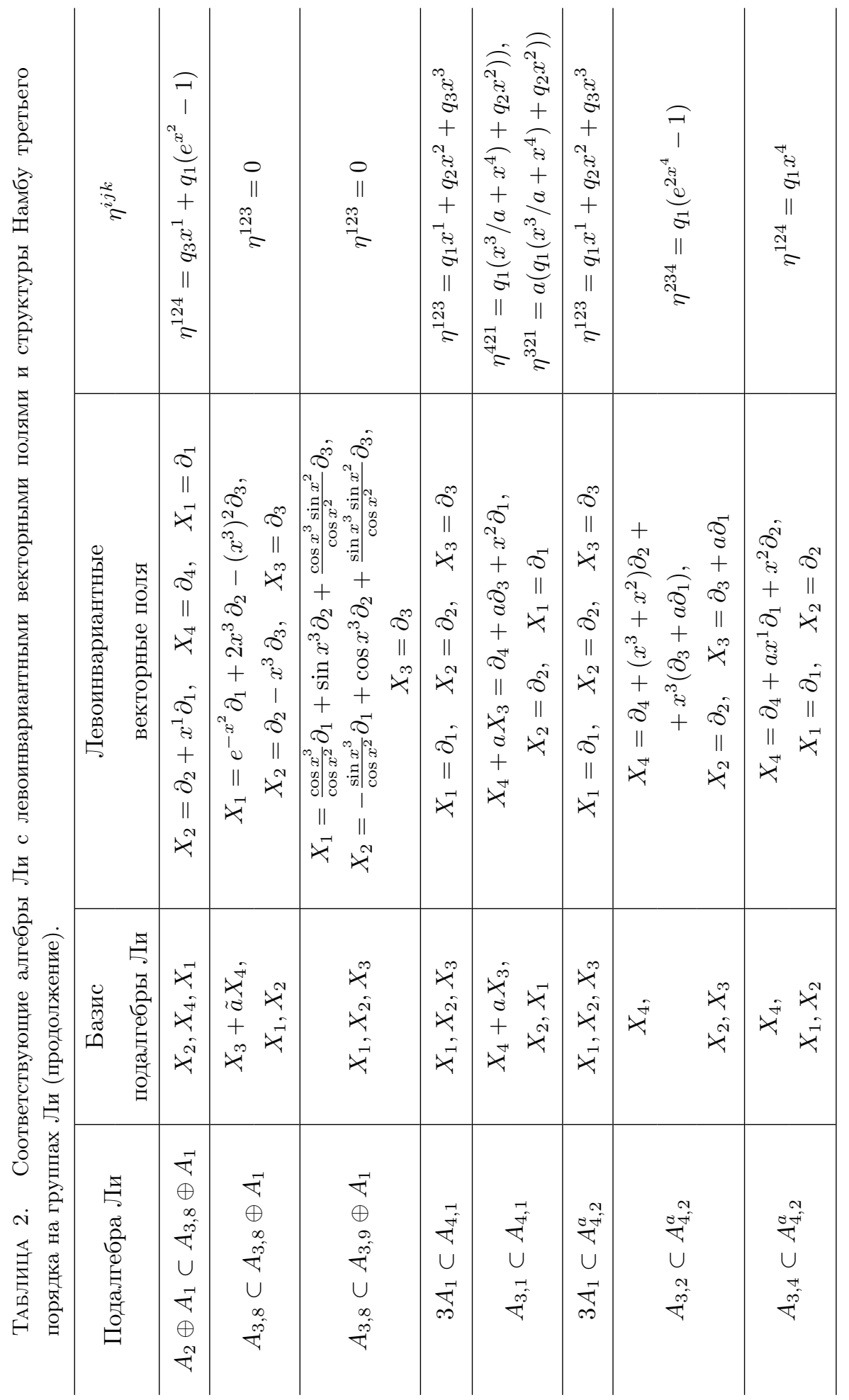




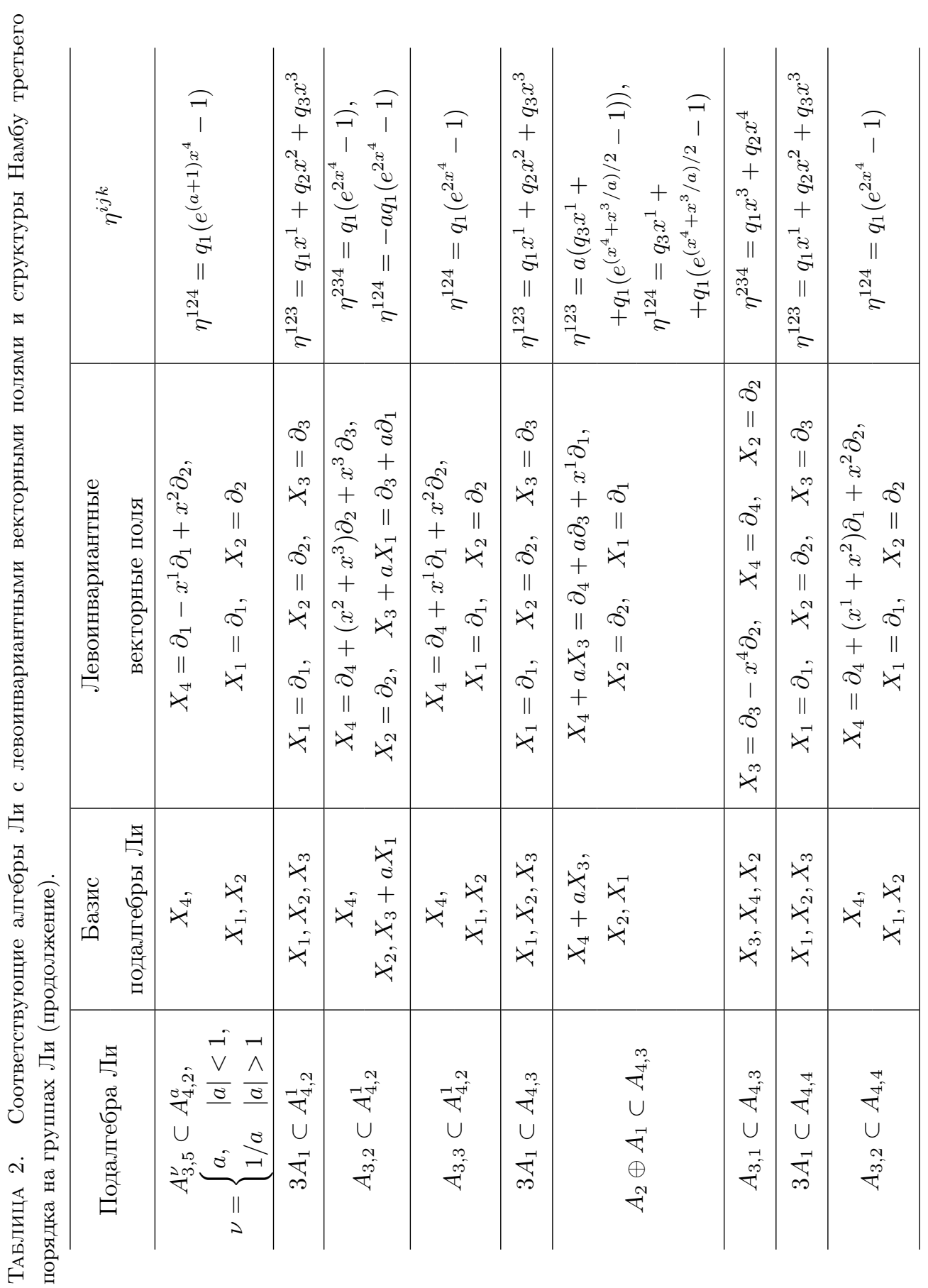




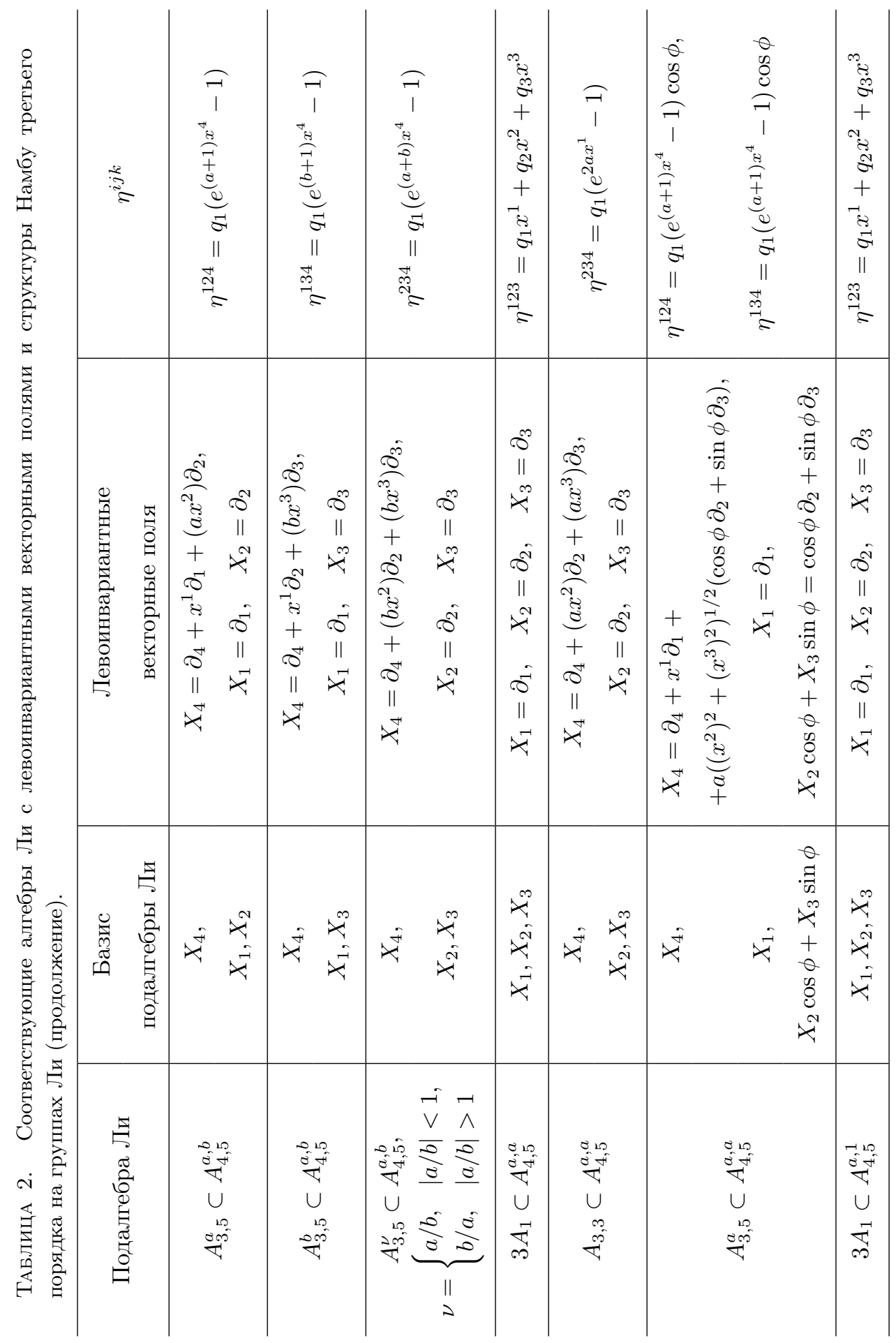




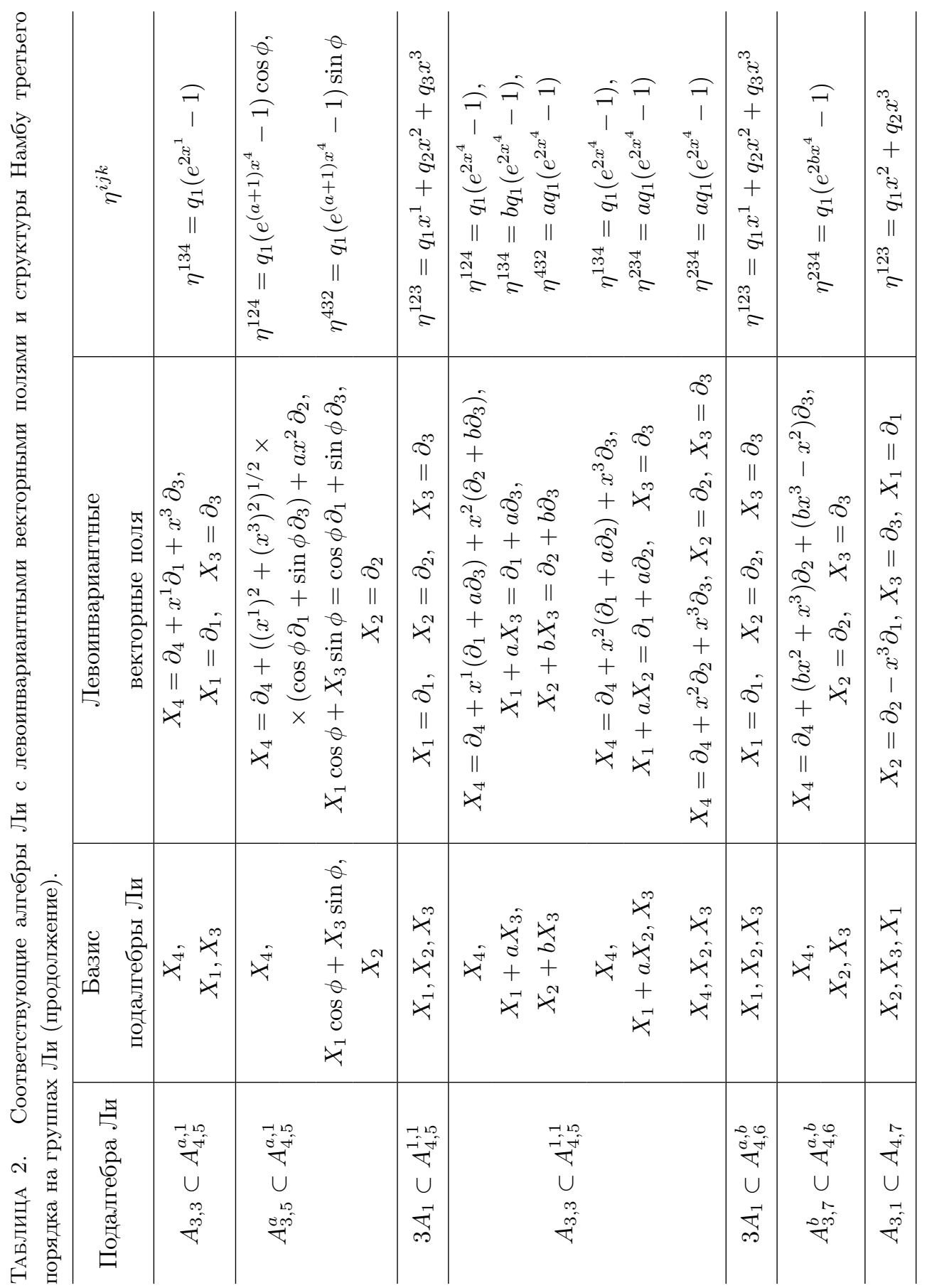




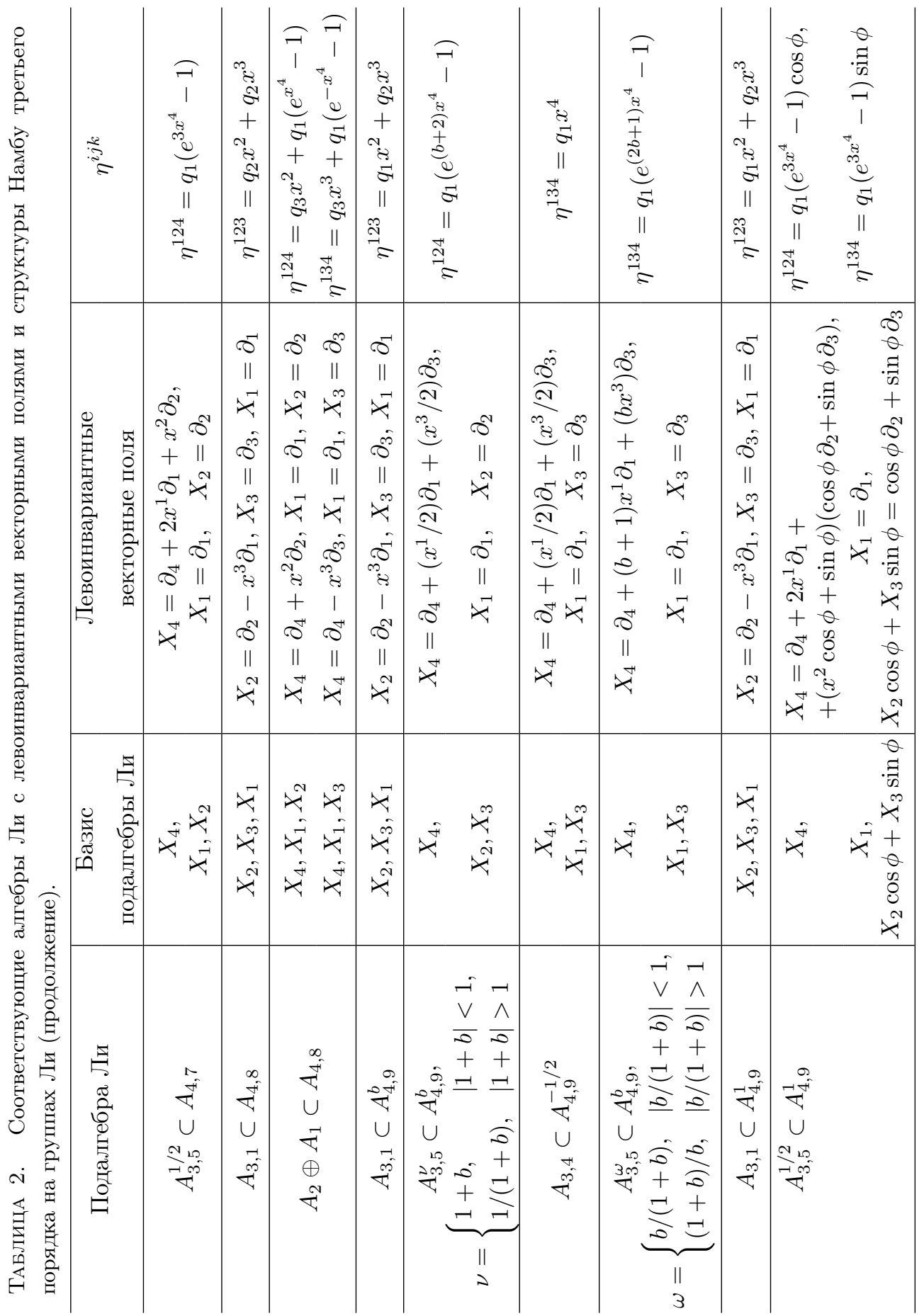




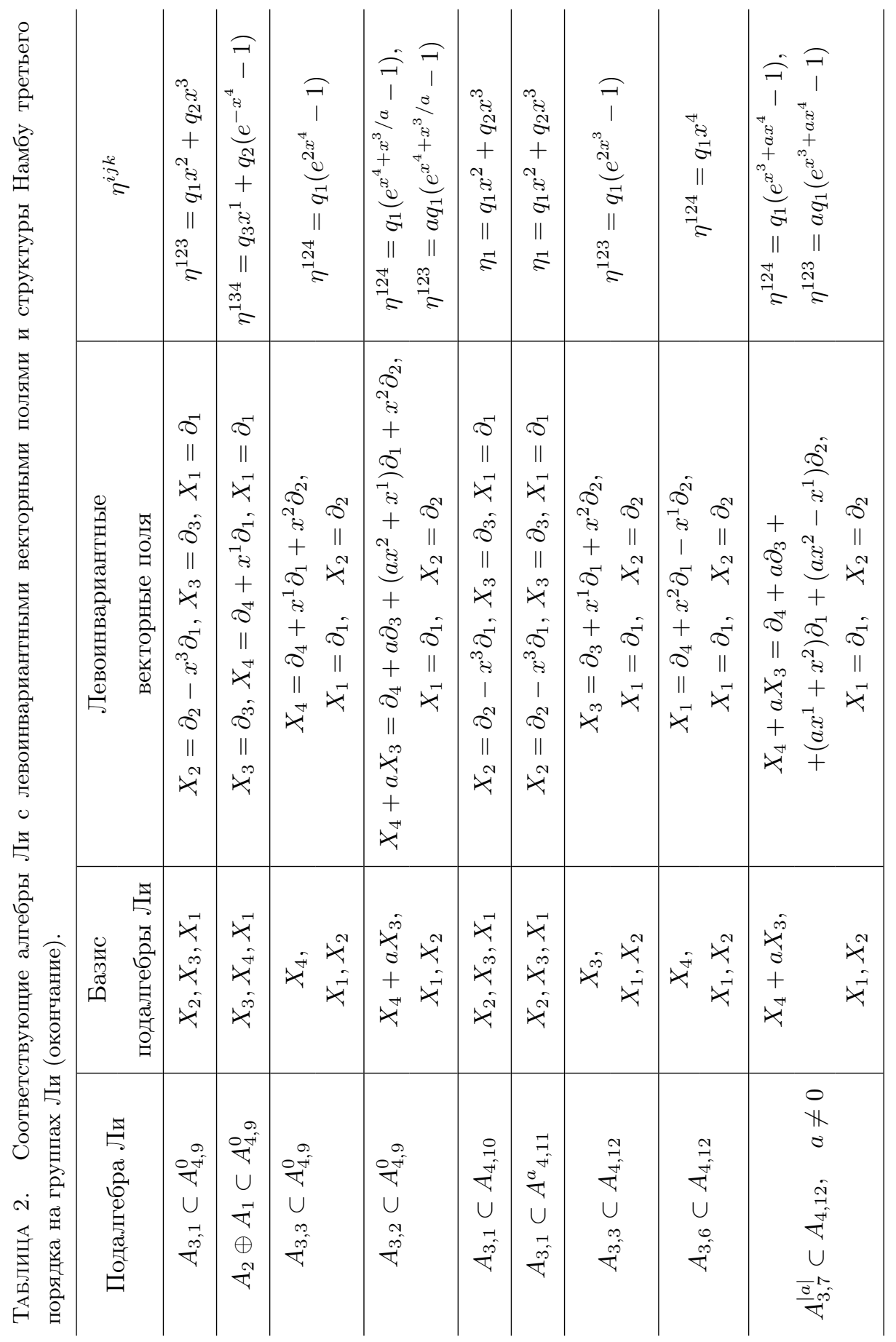


Благодарности. Мы хотели бы выразить искреннюю признательность В. Барзгару и $\Phi$. Дараби за внимательное чтение статьи и полезные комментарии. Исследование поддержано Azarbaijan Shahid Madani University (грант № 27.d.1518).

\section{Список литературы}

[1] Y. Nambu, Phys. Rev. D, 7:8 (1973), 2405-2412.

[2] L. Takhtajan, Commun. Math. Phys., 160:2 (1994), 295-315, arXiv: hep-th/9301111.

[3] Ph. Gautheron, Lett. Math. Phys., 37:1 (1996), 103-116.

[4] D. Alekseevsky, P. Guha, Acta. Math. Univ. Comenian., 65:1 (1996), 1-9.

[5] R. Ibáñez, M. de León, J. C. Marrero, D. Martín de Diego, J. Math. Phys., 38:5 (1997), $2332-2344$.

[6] J. Grabowski, G. Marmo, J. Phys. A: Math. Gen., 32:23 (1999), 4239-4247, arXiv: math/9902128.

[7] N. Nakanishi, Rev. Math. Phys., 10:4 (1998), 499-510.

[8] J. A. de Azcárraga, A. M. Perelomove, J. C. Pérez Bueno, J. Phys. A: Math. Gen., 29:7 (1996), L151-L157.

[9] J. A. de Azcarraga, A. M. Perelomove, J. C. Perez-Bueno, J. Phys. A: Math. Gen., 29:24 (1996), 7993-8009, arXiv: hep-th/9605067.

[10] J. A. de Azcárraga, J. M. Izquierdo, J. C. Pérez Bueno, J. Phys. A: Math. Gen., 30:18 (1997), L607-L616.

[11] R. Ibáñez, M. de León, J. C. Marrero, D. Martín de Diego, Rep. Math. Phys., 42:1-2 (1998), 71-90.

[12] J. Grabowski, G. Marmo, Differ. Geom. Appl., 12:1 (2000), 35-50.

[13] I. Vaisman, J. Lie Theory, 10:1 (2000), 181-194.

[14] N. Nakanishi, "Nambu-Poisson tensors on Lie groups", Poisson Geometry (Warsaw, August 3-15, 1998), Banach Center Publications, 51, eds. J. Grabowski, P. Urbanski, Polish Acad. Sci., Warsaw, 2000, 243-249.

[15] N. Nakanishi, RIMS Kokyuroku, 1504 (2006), 54-59.

[16] G. Ovando, Rev. Un. Mat. Argentina, 45:2 (2004), 55-67, arXiv: math/0309146.

[17] J. Patera, P. Winternitz, J. Math. Phys., 18:7 (1977), 1449-1455.

[18] B. Mojaveri, A. Rezaei-Aghdam, Internat. J. Modern Phys. A, 27:7 (2012), 1250032, 32 pp., arXiv: 1106.1795.

[19] А. В. Болсинов, А. Т. Фоменко, Интегрируемъе гамильтоновы системы. Геометрия, топология, классификация, Удмурт. ун-т, Ижевск, 1999.

[20] P. Tempesta, A. V. Turbiner, P. Winternitz, J. Math. Phys., 42:9 (2001), 4248-4257, arXiv: hep-th/0011209.

[21] T. Curtright, C. Zachos, Phys. Rev. D, 68:8 (2003), 085001, 29 pp., arXiv: hep-th/0212267.

[22] J. Patera, R. T. Sharp, P. Winternitz, H. Zassenhaus, J. Math. Phys., 17:6 (1976), 986-994.

[23] R. O. Popovych, V. M. Boyko, M. O. Nesterenko, M. Lutfullin, J. Phys. A: Math. Gen., 36:26 (2003), 7337-7360, arXiv: math-ph/0301029.

[24] J. Abedi-Fardad, A. Rezaei-Aghdam, Gh. Haghighatdoost, J. Math. Phys., 55:5 (2014), 053507, 11 pp., arXiv: 1401.2533.

Поступила в редакцию 14.07.2014, после доработки 8.09.2014 